\title{
Lung complication in COVID-19 convalescence: A spontaneous pneumothorax and pneumatocele case report
}

\author{
Lorenzo Rampa $^{1 *}$, Antonio Miceli ${ }^{2}$, Francesco Casilli ${ }^{3}$, Tullio Biraghi ${ }^{4}$, Baronio Barbara ${ }^{3}$ and Francesco Donatelli $^{5}$ \\ ${ }^{1}$ Cardio-Thoracic-Vascular Department, San Raffaele Scientific Institute, Milan, Italy \\ ${ }^{2}$ Minimally Invasive Cardiac Department, Istituto Clinico Sant'Ambrogio, Milan, Italy \\ ${ }^{3}$ Cardiology Department, Istituto Clinico Sant'Ambrogio, Milan, Italy \\ ${ }^{4}$ Thoracic Surgery Department, Istituto Clinico Sant'Ambrogio, Milan, Italy \\ ${ }^{5}$ Chair of Cardiac Surgery, University of Milan, Milan, Italy
}

\begin{abstract}
An outbreak of novel coronavirus $(2019-\mathrm{nCoV})$ that began in Wuhan, China, has spread rapidly all over the world, becoming pandemic according WHO on March 11, 20201. The typical symptoms of COronaVIrus Disease 19 (COVID-19) can range from mild influenza like syndrome to severe respiratory illness. The elderly population, especially with comorbidities like chronic bronchitis, emphysema, heart failure, or diabetes, is more likely to develop serious illness. Radiographic findings in acute phase of SARS CoV-2 bilateral interstitial pneumonia have been described in several studies. Pilot Computerized Tomography scan studies show lung abnormalities usually reabsorb in 3 weeks without sequelae. Nevertheless, no large studies have done on severity-based chronic lung injury and late pulmonary complications in recovered patients. Pneumothorax and pneumatocele can be two severe lung complications in acute phase and intubated patients, but there is no evidence they can occur during convalescence. We report the first case of a spontaneous pneumothorax due to traumatic (sneeze related) pneumatocele, occurred after recovery from bilateral interstitial pneumonia SARS-Cov-2.
\end{abstract}

\section{Case report}

A Hispanic 43-year-old man presented with 10-day history of cough and remittent fever treated with Amoxicillin/Clavulanic acid plus Paracetamol by his general practitioner. Due to persistence of fever and resting dyspnea onset, he was admitted in our hospital, presenting also with headache, anosmia, loss of taste and myalgia.

He was a healthy man without any presumed disease, apart from his mild history of smoke until 3 years ago and occasional asbestos exposition related to his job as aerial fitter. He denied family contacts or vaccination for Tuberculosis. The nasopharyngeal swab was positive for SARS CoV-2 and CT scan described extensive bilateral ground glass opacities, especially in middle sections, involving $40 \%$ of lung parenchyma, moreover some areas of consolidation with crazy paving pattern. No emphysema or cysts were observed (Figure 1).

The patient was treated with oxygen mask (maximum $\mathrm{FiO}_{2} 40 \%$ ) achieving good level of oxygen saturation ( $>95 \%)$. Moreover, he started Idroxicloroquine $200 \mathrm{mg}$ bid and Darunavir $800 \mathrm{mg} /$ Cobicistat 150 $\mathrm{mg}$ once a day, Azytromicine $500 \mathrm{mg}$ OD, Ceftriaxone $2 \mathrm{~g}$ OD and enoxaparin 6000 UI OD. Monitoring Chest X rays showed a mild improvement of bilateral pulmonary lesions, and no pneumothorax. After 17 days, he was weaned from oxygen and clinical symptoms ceased, but he could not guarantee home quarantine. He was transferred to rehabilitation hospital and was declared healed from SARS CoV2 infection after two negative nasal swabs. During his rehabilitation, patient presented with sudden dyspnea, hemoptysis and middle back pain after holding back a sneeze. Chest X-ray showed pneumothorax surrounding left lung and a new cavity with thickened walls in left upper lobe. Some air entrapment was also described between neck muscles (Figure 2).
Patient parameters remained stable over time, along with inflammatory markers, but symptoms became more severe, with dyspnea relapse, desaturation to $92 \%$ in air and coagula in sputum for several days. After 30 days, CT scan showed a cavitation in left upper lobe $(10 \times 6,7 \times 6 \mathrm{~cm})$ with thicken wall and hydro-air level, without evident bronchial leaks. No pneumothorax was found surrounding left lung and ground glass opacities were persistent in $25 \%$ of lung volume. According to radiologist, hydro-pneumatocele or abscess cavity were the two potential diagnosis (Figure 3).

In this regard, patient was again transferred to our department, and a CT guided drainage of serum with coagula and air suction was performed. Prophylactic antibiotic therapy with ceftriaxone $2 \mathrm{~g}$ once a day was started. Coltural exams did not show any infection, and cytologic assessment showed only serum liquid and blood cells. Patient remained stable after invasive procedure, with resolution of pain and persistent mild dyspnea. Quantiferon test for Tuberculosis resulted negative. CT scan performed after 36 days showed persistence of cavitation and some grade of resolution of ground glass opacities. Patient was discharged home with diagnosis of hydro-pneumatocele in SARS CoV-2 pneumonia. After 60 days from symptoms onset, patients referred persistence of effort dyspnea and asthenia. Follow up CT scan was performed, demonstrating persistence of ground glass opacities in $20 \%$ of lung parenchyma, diffuse fibrous stripe, and cavity size halving with clot inside (Figure 4).

${ }^{\star}$ Correspondence to: Lorenzo Rampa, Cardio-Thoracic-Vascular department, San Raffaele Scientific Institute, Milan, Italy, Tel: +393924878243, Email: rampa. lorenzo@gmail.com

Received: April 27, 2020; Accepted: May 11, 2020; Published: May 18, 2020 


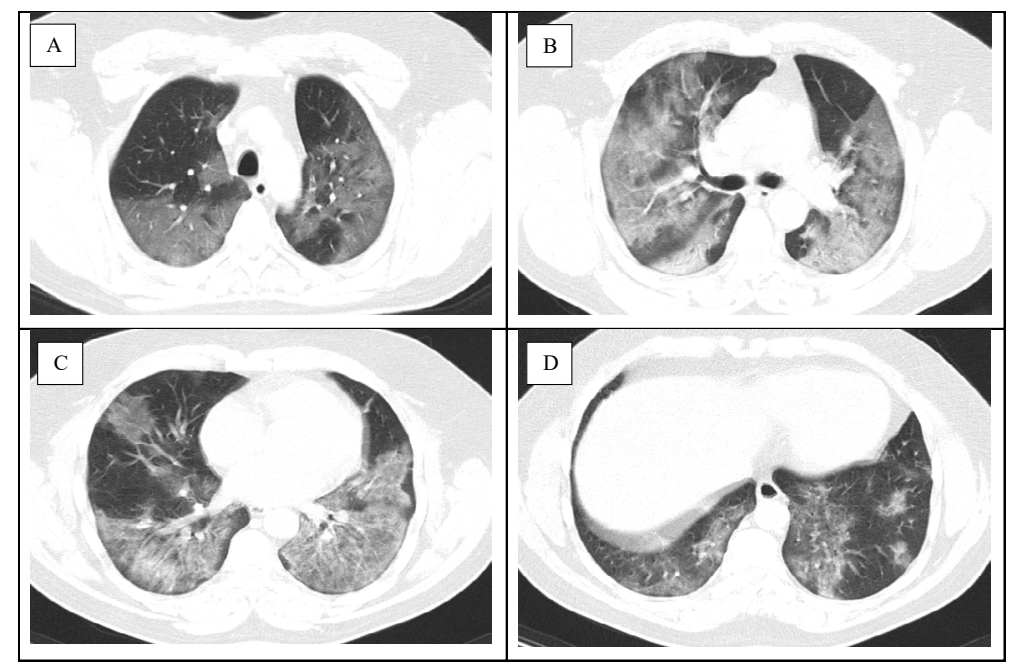

Figure 1. Admission CT scan: Axial CT images obtained at patient presentation showed diffuse ground-glass opacities, involving $40 \%$ of lung parenchyma. Panel A showed diffuse bilateral confluent and patchy ground-glass areas involving upper lobes. Panel B and C included consolidative pulmonary opacities in middle posterior parenchyma areas, and interstitial septal thickening making some areas of crazy paving pattern. Panel D showed less basal bilateral opacities in the basis.

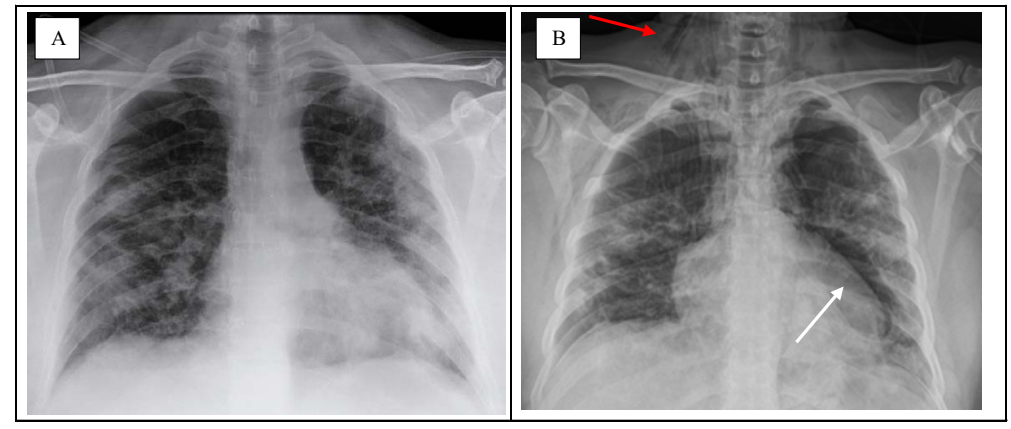

Figure 2. Comparison of Chest X-ray before (Panel A) and after (Panel B) complication onset; Chest X-ray images obtained from the same patient before discharge from hospital (Panel A) and at rehabilitation clinic after symptoms onset (Panel B); Panel A showed bilateral peripheral opacities, mainly in intermediate and basal portions. Panel B elucidated mild improvement in right lung opacities, and a new pneumothorax in apical and middle portion of left lung. A thick-walled cavity within left lung appeared (white arrow). Air around neck muscles was also reported (red arrow).
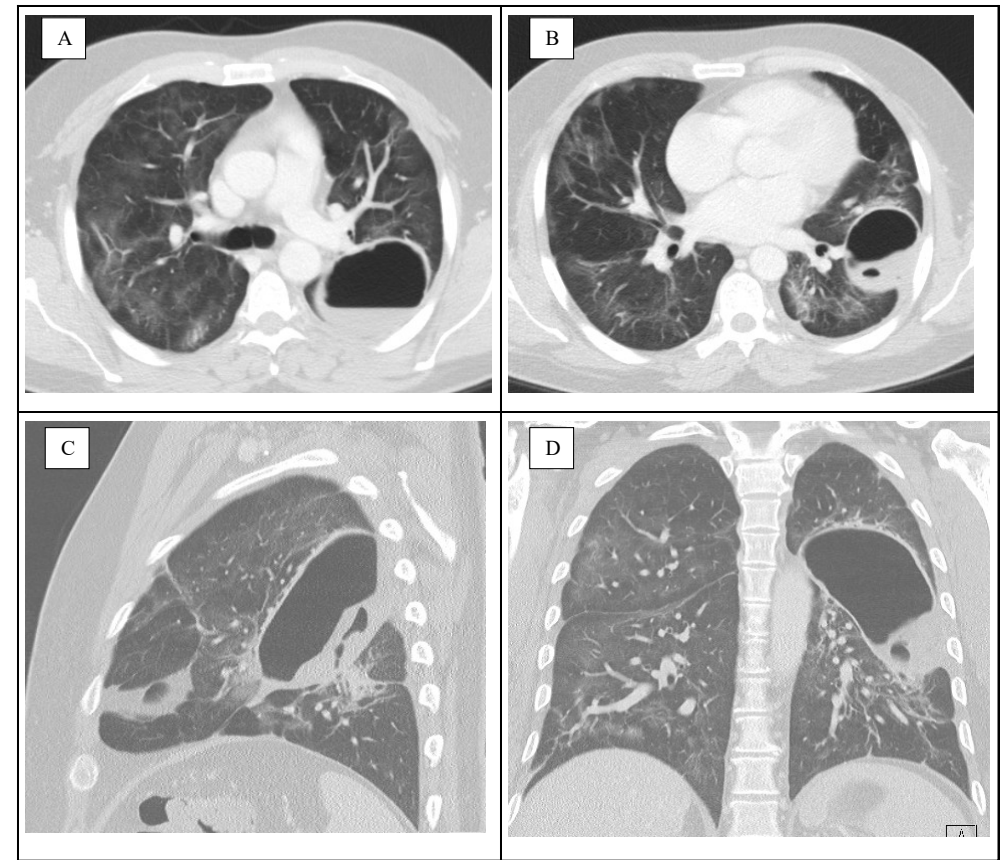

Figure 3. CT scan of Hydro-air pneumatocele: Axial CT images (Panels A and B) obtained after dyspnea and hemoptysis onset showed a thick-walled abnormal gas and liquid-filled cavity close to fissure inside upper left lobe. Diffuse ground-glass opacities was decreased. A sagittal CT image (Panel C) showed pneumatocele with hydro-air level and organized material inside. A coronal CT image (Panel D) elucidated position and extension of cavitation. 


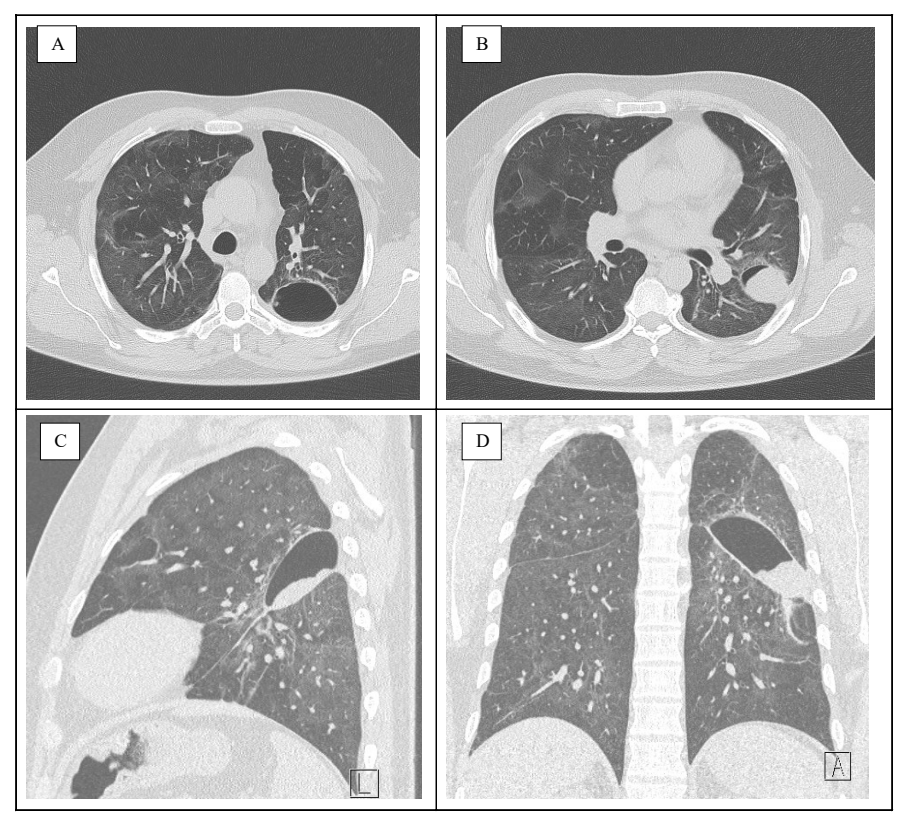

Figure 4. 55 days follow up CT scan: Axial CT images (Panels A and B) obtained after 55 days showed reduction of cavity size. Diffuse ground-glass opacities are still persistent in $20 \%$ of parenchyma.

A sagittal CT image (Panel C) showed organized hydro-air level. A coronal CT image (Panel D) elucidated decreased extension of cavitation.

\section{Comment}

We describe a case of COVID-19 recovered patient who developed spontaneous pneumothorax due to barotraumatic hydropneumatocele. COVID-19 presentation can rarely occur with pneumothorax, accounting around $1 \%$ of radiological findings [1], arriving at 5,9\% in critically ill patients [2]. Cavity may be the result of several pathological processes [3] including suppurative necrosis (e.g., pyogenic lung abscess), caseous necrosis (e.g., tuberculosis), ischemic necrosis (e.g., pulmonary infarction or gangrene), displacement of lung tissue by cystic structures (e.g., Echinococcus), cystic dilatation of lung structures (e.g., ball valve obstruction and Pneumocystis pneumonia) or high pressure traumas. Patients with acute lung distress syndrome are more susceptible to pneumatocele and pneumothorax, usually due to subpleural bullas rupture caused by hyper oxygenation and high pressure ventilation injury [4].

According to patient's clinical features, we excluded lung intrinsic diseases and ventilatory trauma in ARDS. Suppurative process and fungal infection were ruled out because patient did not develop fever, inflammation markers were normal and fluid drainage reveled no pathogen growth. A recent case report has shown the appearance of giant bullas and subpleural emphysemas with secondary pneumothorax in severe case of COVID-19 during the acute phase, not related to invasive intubation [5]. Interesting, our patients didn't require these supports, and CT scan at the admission did not show any sign of pulmonary emphysema or bubbles that could justify secondary pneumothorax. Moreover, in our case, complication occurred after patient recovery, suggesting persistence of lung structural frailty even after disappearance of symptoms and inflammation marker normalization. Histologic and autoptic studies in lungs of COVID-19 people died for severe respiratory distress revealed a mix of diffuse alveolar damage and capillary injury, along with severe organizing hemorrhagic pneumonitis. An important fibrin deposition within septal capillary lumens and walls and endothelial cell necrosis described a thrombotic necrotizing capillary injury syndrome [6]. According to this findings, it's reasonable that alveoli structure may be frail, and septal rupture can occur even after mild injury.

A barotrauma related to a sneeze could have led to a wild rupture in interstitial septa inside lung parenchyma, weakened by hyperinflammation, and thanks to a preserved compliance of the lung, air spread into pleural cavity, causing pneumothorax. A bronchus communication enhanced hemoptysis and growth of pneumatocele dimension, without a check valve mechanism liable of complete lung collapse. On the other hand, no invasive treatment was required for acute scenario, and pneumothorax spontaneously resolved. We concluded for traumatic pneumothorax due to hydro-pneumatocele in a SARS-CoV2 recovered pneumonia.

\section{Acknowledgments}

We thank the patient described in case report to give us the consent and share his clinical information.

\section{Disclosure}

All the authors had full access to all the data in the study and takes responsibility for the integrity of the data and the accuracy of the paper.

\section{Conflict of interest}

All authors declare no conflict of interest. No prior or related publications have been done on the topic.

\section{References}

1. Chen N, Zhou M, Dong X, Jieming Q, Gong F, et al. (2020) Epidemiological and clinical characteristics of 99 cases of 2019 novel coronavirus pneumonia in Wuhan, China: a descriptive study. Lancet 395: 507-513. [Crossref]

2. Yao W, Wang T, Jiang B, Gao F, Wang L, et al. (2020) Emergency tracheal intubation in 202 patients with COVID-19 in Wuhan, China: lessons learnt and international expert recommendations. Br J Anaesth 125: e28-e37. [Crossref]

3. Gadkowski LB, Stout JE (2008) Cavitary Pulmonary Disease. Clin Microbiol Rev 221 305-333. [Crossref]

4. Ware LB, Matthay MA (2000) The Acute Respiratory Distress Syndrome. $N$ Engl $J$ Med 342: 1334-1349. [Crossref]

5. Sun R, Liu H, Wang X (2020) Mediastinal Emphysema, Giant Bulla, and Pneumothorax Developed during the Course of COVID-19 Pneumonia. Korean J Radiol 21: 541 [Crossref]

6. Magro C, Mulvey JJ, Berlin D, Nuovo G, Salvatore S, et al. (2020) Complement associated microvascular injury and thrombosis in the pathogenesis of severe COVID-19 infection: a report of five cases. Transl Res 220: 1-13. [Crossref]

Copyright: (C2020 Rampa L. This is an open-access article distributed under the terms of the Creative Commons Attribution License, which permits unrestricted use, distribution, and reproduction in any medium, provided the original author and source are credited. 